\title{
High throughput DNA sequencing to detect differences in the subgingival plaque microbiome in elderly subjects with and without dementia
}

\author{
Andrew F Cockburn ${ }^{1}$, Jonathan M Dehlin ${ }^{1}$, Tiffany Ngan ${ }^{2}$, Richard Crout ${ }^{2}$, Goran Boskovic ${ }^{3}$, James Denvir ${ }^{3}$, \\ Donald Primerano ${ }^{3}$, Brenda L Plassman ${ }^{4}$, Bei Wu ${ }^{5}$ and Christopher F Cuff ${ }^{*}$
}

\begin{abstract}
Background: To investigate the potential association between oral health and cognitive function, a pilot study was conducted to evaluate high throughput DNA sequencing of the V3 region of the $16 \mathrm{~S}$ ribosomal RNA gene for determining the relative abundance of bacterial taxa in subgingival plaque from older adults with or without dementia.

Methods: Subgingival plaque samples were obtained from ten individuals at least 70 years old who participated in a study to assess oral health and cognitive function. DNA was isolated from the samples and a gene segment from the V3 portion of the $16 \mathrm{~S}$ bacterial ribosomal RNA gene was amplified and sequenced using an Illumina HiSeq1000 DNA sequencer. Bacterial populations found in the subgingival plaque were identified and assessed with respect to the cognitive status and oral health of the participants who provided the samples.

Results: More than two million high quality DNA sequences were obtained from each sample. Individuals differed greatly in the mix of phylotypes, but different sites from different subgingival depths in the same subject were usually similar. No consistent differences were observed in this small sample between subjects separated by levels of oral health, sex, or age; however a consistently higher level of Fusobacteriaceae and a generally lower level of Prevotellaceae was seen in subjects without dementia, although the difference did not reach statistical significance, possibly because of the small sample size.
\end{abstract}

Conclusions: The results from this pilot study provide suggestive evidence that alterations in the subgingival microbiome are associated with changes in cognitive function, and provide support for an expanded analysis of the role of the oral microbiome in dementia.

Keywords: Cognitive impairment, Oral disease, Oral microbiome, Subgingival plaque

\section{Background}

In addition to the hypothesized link between oral health and chronic systemic diseases, such as cardiovascular disease, stroke, and diabetes, there now appears to be an association between oral health and neurodegenerative diseases, ranging from mild to moderate loss of cognitive function to Alzheimer's Disease (AD) [1]. Poorer cognitive performance and tooth loss have been linked

\footnotetext{
* Correspondence: ccuff@hsc.wvu.edu

'Microbiology, Immunology \& Cell Biology, School of Medicine, Robert C. Byrd Health Sciences Center, West Virginia University, P.O. Box 4622 Morgantown, WV 26506-4622, USA

Full list of author information is available at the end of the article
}

epidemiologically in both retrospective and prospective studies [2-7], and tooth loss has been associated with an increased risk of both dementia and cognitive decline [8]. Indeed, increasing tooth loss over time is associated with increased likelihood of low cognitive scores [2]. Beyond epidemiological associations, independent lines of experimental evidence support the hypothesis that bacteria associated with diseases of the oral cavity contribute to neurodegeneration. Oral bacteria and bacteria closely related to those found in the oral cavity have been found at a higher frequency post mortem in the brains of patients with $\mathrm{AD}$ than in those of patients who did not have AD $[9,10]$. In addition, the Third National
Ciomed Central

(C) 2012 Cockburn et al.; licensee BioMed Central Ltd. This is an Open Access article distributed under the terms of the Creative Commons Attribution License (http://creativecommons.org/licenses/by/2.0), which permits unrestricted use, distribution, and reproduction in any medium, provided the original work is properly cited. 
Health and Nutrition Examination Survey (NHANESIII) provided evidence that gingival bleeding and loss of periodontal attachment were associated with lower cognitive function [11]. Furthermore, subjects with high levels of antibody against the periodontal pathogen Porphyromonas gingivalis had significantly greater impaired verbal memory and subtraction test performance, and this finding remained robust when adjusting for potential sociodemographic and vascular confounders [12]. Levels of immunoglobulin G (IgG) antibodies and serum tumor necrosis factor levels have been found to discriminate between normal subjects and AD patients [7], and recently Sparks Stein et al. [13] found that elevated antibody levels to periodontal disease bacteria were observed in subjects years before cognitive impairment, suggesting that periodontal disease could potentially contribute to the risk of $\mathrm{AD}$ onset or progression.

If oral health is linked to neurodegeneration, it is plausible that bacteria found in the oral cavity play a causal role in establishing this link. The collection of microorganisms in the oral cavity, the 'oral microbiome', has been studied using a variety of molecular methods that can identify both cultivatable and non-cultivatable bacteria within various ecological niches in the oral cavity $[14,15]$. Despite the evidence linking oral health and cognitive function, there is a paucity of empirical data that assess the oral microbiome in patients with cognitive degeneration [16]. Microbiome analysis could be used to determine whether the bacterial compositions of the oral microbiome or 'bacterial signatures' could serve as a predictive biomarker for increased risk of cognitive impairment. It is also possible that preventive treatment could target the makeup of the oral microbiome and the efficacy of such treatment could be monitored with this approach.

Recent advances in the availability and reduced costs of high throughput DNA sequencing and bioinformatics tools provide a broadly available and increasingly cost effective method to identify bacterial populations found in polymicrobial biofilms associated with human tissue, including the oral cavity [17-19]. The present study was undertaken to develop a sampling and analysis pipeline using next generation DNA sequencing technology that could be used to characterize microbial populations in subgingival plaque samples. Using an Illumina HiSeq1000 DNA sequencer and a sample preparation and analysis pipeline that enabled multiple samples to be sequenced within the same sequencing lane, we were able to generate and analyze economically more than one million bacterial DNA sequences from each of 15 subgingival plaque samples. The participants were enrolled in a study that assessed oral health and cognitive function among adults at least 70 years old from West Virginia, some of whom were from medically underserved communities [20].
These sequences were analyzed using bioinformatics tools available in the publicly accessable software package Quantitative Insights into Microbial Ecology (QIIME) [21], and sequence comparisons were made among participants who were clinically assessed as normal or exhibited alterations in cognitive function. The results provide a road map for future efforts to use high throughput DNA sequencing to characterize the oral microbiome in the context of systemic disease, and provide preliminary evidence that differences exist in the bacterial composition of subgingival plaque in patients with alterations in cognitive function. In contrast to other approaches to microbiome analysis, high throughput sequencing holds out the promise of also being useful for metagenomic analysis of the oral microbiome to identify potential virulence factors that contribute to systemic disease.

\section{Methods}

\section{Oral health screening, cognitive analysis, and sample collection}

All samples were collected under a protocol reviewed and approved by the West Virginia University Institutional Review Board. The criteria for study participants were age 70 years or older, resident of West Virginia, communityliving, and at least four natural teeth. Oral evaluations were performed by calibrated researchers using guidelines from the NHANES 1999 to 2000 [22]. A psychometrician administered to the participants a battery of neuropsychological measures that assessed verbal and visual memory, language, executive function, orientation, praxis, and reading ability. Depression was assessed using the Geriatric Depression Scale [23]. A proxy informant, usually a spouse or adult child, provided information about the participant's cognitive function, functional limitations, medical history, and medications. All collected data were reviewed by two study psychologists and diagnoses were assigned within three cognitive categories: normal cognitive function, cognitive impairment without dementia (CIND), and dementia. The Diagnostic and Statistical Manual of Mental Disorders, Fourth Edition, criteria were used for the diagnosis of dementia [24]. CIND was defined as mild cognitive or functional impairment reported by the participant or informant that did not meet the criteria for dementia, or performance on neuropsychological measures that was both below expectations based on reading ability and educational and occupational history, and at least 1.5 standard deviations below published norms on any test within a cognitive domain (for example, memory, orientation, language, executive function, praxis). Diagnoses were anchored by these criteria, but the final diagnoses were based on clinical judgment. Similar assessment and diagnostic procedures have been used and validated in multiple large epidemiological studies on cognitive impairment in later life $[25,26]$. 
A total of 15 samples of bacterial DNA from 10 individuals were sequenced for this report, four of which (N1, N2, C2, C3) were obtained and partially analyzed during an earlier phase of the study [16]. Eleven additional subgingival plaque samples were obtained using sterile periodontal curettes from pocket probing depths of 1 to $3 \mathrm{~mm}, 3$ to $5 \mathrm{~mm}$, or $>5 \mathrm{~mm}$. These samples were collected into tubes containing Invitek SalivaGene DNA stabilization buffer (STRATEC Molecular GmbH, Berlin, Germany). In most cases, multiple plaque samples from the same pocket probing depth in the same participant were pooled into one tube for DNA extraction.

\section{DNA extraction}

DNA from the 11 new samples was purified using an Invitek PSP SalivaGene DNA Kit. As part of the purification procedure, $100 \mu \mathrm{g}$ of lysozyme (Sigma-Aldrich, St. Louis, MO, USA) was added to each tube, the mixture incubated at $37^{\circ} \mathrm{C}$ for 10 minutes, and then processed according to the manufacturer's recommendations.

\section{PCR and fragment purification}

PCR primers, conditions for amplification of sequences in the V3 region of the $16 \mathrm{~S}$ ribosomal RNA gene, and a multiplexed DNA sequencing strategy were as described in Bartram et al. [27] unless otherwise indicated. The V3 region varies in length by about 30 base pairs among different species of bacteria in the Greengenes database, and the sequences obtained and analyzed in this study showed a similar size variability. The amplicon ranges from 296 to 327 base pairs, of which 160 base pairs is the primer. High pressure liquid chromatographypurified PCR primers were obtained from Integrated DNA Technologies (Coralville, IA, USA). Purified DNA was amplified using an AccuPrime PCR Kit (Invitrogen Life Technologies, Grand Island, NY, USA) on an MJ Research PTC-200 Thermal Cycler using the following conditions: $95^{\circ} \mathrm{C}$ for 6 minutes denature; $95^{\circ} \mathrm{C}$ for 2 minutes, $50^{\circ} \mathrm{C}$ for 2 minutes, $72^{\circ} \mathrm{C}$ for 2 minutes 30 cycles; $72^{\circ} \mathrm{C}$ for 4 minutes extend. Each reaction contained $0.5 \mu \mathrm{l}$ TAQ polymerase, $5 \mu \mathrm{l} 10 \mathrm{x}$ buffer $1(600 \mathrm{mM}$ TrisSO4 (pH 8.9), $180 \mathrm{mM}$ (NH4)2SO4, $20 \mathrm{mM} \mathrm{MgSO} 4$, 2 mM dGTP, 2 mM dATP, 2 mM dTTP, 2 mM dCTP, thermostable AccuPrime ${ }^{\mathrm{TM}}$ protein, 10\% glycerol), $20 \mu \mathrm{M}$ forward primer, $20 \mu \mathrm{M}$ reverse primer, and up to $60 \mathrm{ng}$ DNA in a total volume of $50 \mu \mathrm{l}$. PCR reactions were performed in triplicate and reaction products were pooled prior to purification. Because there was a low concentration of DNA in some of the samples, it was necessary to perform 30 cycles of amplification to obtain sufficient material to view on a gel. Pooled PCR products were purified by electrophoresis through $2 \%$ agarose in Tris/ borate/EDTA gels and the bands corresponding to approximately 300 base pairs were excised and purified using a QIAquick Gel Extraction Kit (Qiagen, Valencia, CA, USA) according to the manufacturer's directions.

\section{DNA sequencing}

Indexed libraries were pooled so that 12 libraries were sequenced in each lane of the flow cell. Eight pmols of the pooled libraries were clustered onto an Illumina v2 sequencing flow cell using an Illumina cBOT. Libraries were then sequenced in a $2 \times 125$ bp paired-end strategy on an Illumina HiSeq1000, so that the forward and reverse reads could be assembled into a single contig. Reads were converted from Illumina bcl format to fastq format and separated into bins based on exact match to the index using CASAVA 1.8.2 (Illumina, San Diego CA, USA). An average of 6.7 million reads/sample passing the filter was sequenced in each library.

\section{DNA sequence processing}

Sequence files were initially processed by removing sequences corresponding to linkers and primers by automated batch processing using scripts written in-house. In an effort to reduce artifacts generated by sequencing errors, a strict quality filtering protocol was employed that reduced the number of analyzed sequences to approximately $35 \%$ of the total number of sequences generated. Nevertheless, an average of more than two million high quality reads was obtained from each sample. Quality filtering of DNA sequences was performed using the following steps: 1) Sequences were first filtered by the Illumina software to eliminate the poorest reads (Q score $\geq 30$ ) and imperfect primer matches. 2) The forward and reverse sequences were matched to construct a sequence that spanned the entire region between the primers with a program written in-house. The original Illumina sequences were all 125 bases in length, which is where the run ended. The pairing strategy overlaid the two 3' ends starting with an overlap of 58 bases. The overlap window was extended one base at a time to 89 bases until a perfect match was obtained in the overlap region. Any pair of sequences that did not match at $100 \%$ identity in any of the size windows was discarded. This step eliminated $56 \%$ of the sequences, which overwhelmingly had the lowest quality scores. In general, the sequence quality was better in the middle than at the end, so this preferentially eliminated sequences with sequencing artifacts. 3) Paired sequences with a Phred quality score of less than five were discarded. This removed a few remaining low quality sequences, especially any that had low quality in the regions between the primers and the overlap. 4) The sequences were clustered by matching against the Greengenes database, which is a curated collection of known bacterial $16 \mathrm{~S}$ sequences. Sequences that did not match any of the known bacterial sequences with $97 \%$ identity were 
discarded. This removed chimeras and most major PCR artifacts and represented approximately $5 \%$ of the total remaining sequences. Matching at $97 \%$ identity meant that any single base PCR artifacts would be combined with the corresponding authentic sequence (since the region is about 100 bases long, up to three single base changes will be ignored). 5) The resulting table of operational taxonomic units (OTUs) was filtered to remove any sequences that appeared less than 150 times.

Finally, scripts written in-house in biopython were used to convert the filtered Illumina data to the FASTA format for analysis by QIIME for taxonomic assignment and measurements of microbial diversity, but scripts to do this are now part of QIIME. To process Illuminagenerated files in QIIME, the file headers were changed to begin with '>sample_number' where 'sample' is the sample number and 'number' is the number of the sequence in the file. All of the sequences were then combined into one file for analysis with QIIME. DNA sequences generated and analyzed in this study can be found at the National Center for Biotechnology Information Sequence Read Archive, project number SRA057340.

\section{QIIME}

All QIIME analyses were performed on a virtual server hosted by Amazon Web Services using an existing QIIME image. The server had the following specification: QIIME 1.4.0 EBS East XLARGE (ami-438d5b2a). The following QIIME scripts were used during analysis and default parameters were used unless otherwise noted: 1) 'pick_ reference_otus_through_otu_table.py' matched sequences at $97 \%$ sequence identity with OTUs associated with specific bacterial phylotypes in the Greengenes database (4Feb2011); 2) 'summarize_taxa_through_plots.py' generated bar graphs of the relative abundance of different taxa in each sample; 3) 'alpha_rarefaction.py' generated alpha rarefication plots; 4) 'pick_rep_set.py', 'align_seqs.py', 'filter_alignment.py', and 'make_phylogeny.py' were chained to generate a phylogenetic tree of the OTUs; 5) 'beta_diversity_through_plots.py' (using the phylogenetic tree and the weighted UniFrac option) generated a beta diversity table and principle coordinate plots for the inter-subject diversity; and 6) 'otu_category_significance. py' generated analysis of variance (ANOVA) scores for all OTUs versus various categories. This script calculated raw, Bonferroni corrected, and false discovery rate corrected probabilities.

\section{Statistical analysis}

Data analyses involved logistic regression as implemented in JMP/Pro Software (version 9.0.2) and random forests as implemented in R Software [28].

\section{Results}

Demographics and health status of study participants

A total of 15 samples was obtained from 10 individual participants. Participants ranged from 70 to 101 years old, were a nearly even mix of men and women, and all but one were self-identified as Caucasian. The number of teeth retained by the participants ranged from 7 to 22. Additional features of their oral health examinations are listed in Table 1.

\section{Generation and filtering of DNA sequences}

The Illumina sequencing run of the 15 DNA samples generated a total of more than 100 million paired-end DNA sequencing reads that met the initial quality filtering criteria (Table 2). Approximately 44 million reads could be aligned to produce a continuous sequence with at least a 58 base overlap with $100 \%$ identity and of these, more than 34 million DNA sequences passed all quality filtering steps. These sequences were clustered into OTUs matching the Greengenes database at $97 \%$ homology. An OTU clustered at $97 \%$ homology was

Table 1 Demographics and health status of study participants

\begin{tabular}{|c|c|c|c|c|c|c|c|c|c|c|}
\hline Sample ID & $\begin{array}{l}\text { Cognitive } \\
\text { assessment }\end{array}$ & Age & Sex & Race & PPD & $\begin{array}{l}\text { Number } \\
\text { of teeth }\end{array}$ & $\begin{array}{l}\text { Gingivitis } \\
\text { score }\end{array}$ & $\begin{array}{l}\text { Plaque } \\
\text { Index }^{a}\end{array}$ & $\begin{array}{l}\text { Number of } \\
\text { coronal caries }\end{array}$ & $\begin{array}{l}\text { Number of } \\
\text { root caries }\end{array}$ \\
\hline N1 & Normal & 77 & $\mathrm{~m}$ & w & 2 & 22 & 0.18 & 0 & 0 & 1 \\
\hline $\mathrm{N} 2$ & Normal & 77 & f & w & 2 & 11 & 0.82 & 0 & 5 & 4 \\
\hline $\mathrm{C} 1$ & CIND & $>90$ & $\mathrm{~m}$ & w & 4 & 20 & 0.00 & 1 & 0 & 0 \\
\hline $\mathrm{C} 2$ & CIND & $>90$ & f & w & 3 & 19 & $N D^{b}$ & 1 & 4 & 3 \\
\hline $\mathrm{C} 3$ & CIND & 74 & f & $b$ & 3 & 22 & 0.73 & 0 & 0 & 0 \\
\hline D1 & Dementia & 82 & $\mathrm{~m}$ & w & 4 & 07 & 0.14 & 0 & 0 & 0 \\
\hline $\mathrm{D} 2$ & Dementia & 78 & f & w & 4 & 28 & 1.00 & 1 & 0 & 0 \\
\hline D3 & Dementia & 89 & f & w & 4 & 14 & 0.71 & 2 & 13 & 3 \\
\hline D4 & Dementia & 70 & $\mathrm{~m}$ & w & 6 & 19 & 1.00 & 3 & 36 & 0 \\
\hline D5 & Dementia & $>90$ & $f$ & w & 2 & 10 & 0.00 & 1 & 2 & 0 \\
\hline
\end{tabular}

${ }^{\mathrm{a}}$ As described in Methods; ${ }^{\mathrm{b}}$ Not done. b, black; CIND, cognitively impaired, without dementia; f, female; m, male; PPD, pocket probing depth (mm); w, white. 
Table 2 Number of DNA sequences obtained during processing

\begin{tabular}{lll}
\hline Processing step & Total & $\%$ \\
\hline Initial Number of Sequences & $101,081,862$ & 100 \\
Successful end pairing & $44,142,704$ & 44 \\
PHRED score $>5$ & $36,213,577$ & 36 \\
OTUs identified and analyzed & $34,655,555$ & 34 \\
Average sequences/sample & $2,310,370$ & n.a. \\
\hline
\end{tabular}

n.a. not applicable; OTU operational taxonomic units.

considered a unique phylotype that approximates a 'species', but the $97 \%$ identity rule typically applies across the entire $16 \mathrm{~S}$ rDNA sequence. If the V3 region is more or less variable this rule may not apply accurately to a shorter read length. An average of about 2.4 million sequences was analyzed per DNA sample. Approximately $34 \%$ of the initial output of DNA sequences could thus be assigned as high quality OTUs for analysis using this sequencing and quality filtering pipeline. OTUs containing fewer than 150 sequences were discarded, resulting in identification of a total of 492 OTUs in this study [See Additional file 1].

\section{Population diversity in samples}

Alpha diversity is the amount of population diversity within a given sample. Alpha diversity was measured as the number of phylotypes observed versus number of sequences analyzed. Once each sequence was assigned to an OTU, QIIME was used to assess the alpha diversity of each subject and generate diversification plots (Figure 1). The number of OTUs identified in subgingival plaque samples ranged from 182 (CIND2) to 385 (Dementia 4). Population diversity essentially plateaued after approximately 350,000 reads were analyzed, and

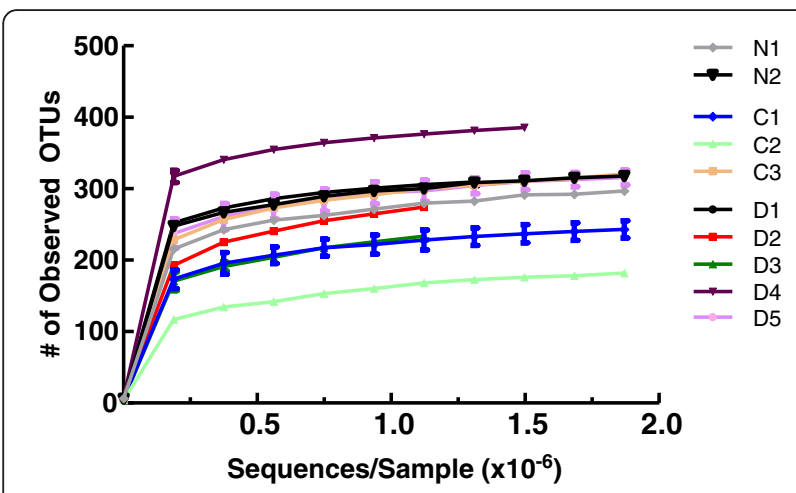

Figure 1 Alpha rarefaction plot demonstrating phylotype diversity in subgingival plaque samples. Shown are the numbers of different Operational Taxonomic Units (OTUs) as a function of the numbers of sequences analyzed and generated with QIIME. OTUs that occur less than 150 times/sample are not included. C, cognitively impaired, not dementia; $\mathrm{D}$, dementia; $\mathrm{N}$, normal. this result was consistent across participants, irrespective of the final diversity. Using the QIIME script otu category_significance.py to run ANOVA, the amount of diversity among participants was not linked to the method of DNA isolation, mental status, age, race, or parameters of oral health listed in Table 1 (data not shown). Although the population diversity varied among participants, multiple samples from individual participants yielded similar curves when analyzed separately. This characteristic is illustrated by the small error bars in Figure 1, where results from separate samples taken from different probing depths from some individuals were combined and averaged.

\section{Taxonomic assignments}

Taxonomic assignments for DNA sequences from each sample were made and analyzed at the phylum, class, order, family, and genus levels. Because of the relatively short region sequenced, assignments at the species level were not robust. Results are shown for analysis of samples at the phylum and family levels for each participant and for multiple probing depth sites where available. In general, most of the identified bacteria were distributed among the phyla Fusobacteria, Bacteroidetes, Firmicutes, TM7, Actinobacteria, and Proteobacteria with less than $1 \%$ contribution of bacteria from any other phyla (Figure 2). There appeared to be a higher proportion of Fusobacteria-specific sequences in the samples from participants who did not have dementia compared to those who did. Additionally, at the phylum level the proportion of sequences identified as members of the Bacteroidetes phylum seemed to be slightly elevated in the samples from subjects with dementia. These potential relationships were explored in more detail at lower taxonomic levels. Sixty-eight different families were identified during taxonomic assignment (Figure 2) and the large interperson variation in bacterial populations became evident. Nevertheless, the most striking observation was that in a comparison of samples in the non-dementia versus the dementia groups, the non-dementia samples had a higher proportion of sequences identified as from the family Fusobacteriaceae (primarily genera Fusobacterium and Leptotrichia) and a lower proportion of sequences from Prevotellaceae (almost entirely Prevotella).

The two most common families varied in the diversity of detected phylotypes. Sixty-nine phylotypes of Prevotella and one rare phylotype not assigned to a genus were seen in the Prevotellaceae. There was substantial diversity in the phylotypes of Prevotella (Figure 3), as has been recently reported in ethnically diverse populations including those of Aboriginal descent in Australia [29] and in a population from the Netherlands [19]. In contrast, only 17 phylotypes of Fusobacteriaceae were found, even though about twice as many sequences were 


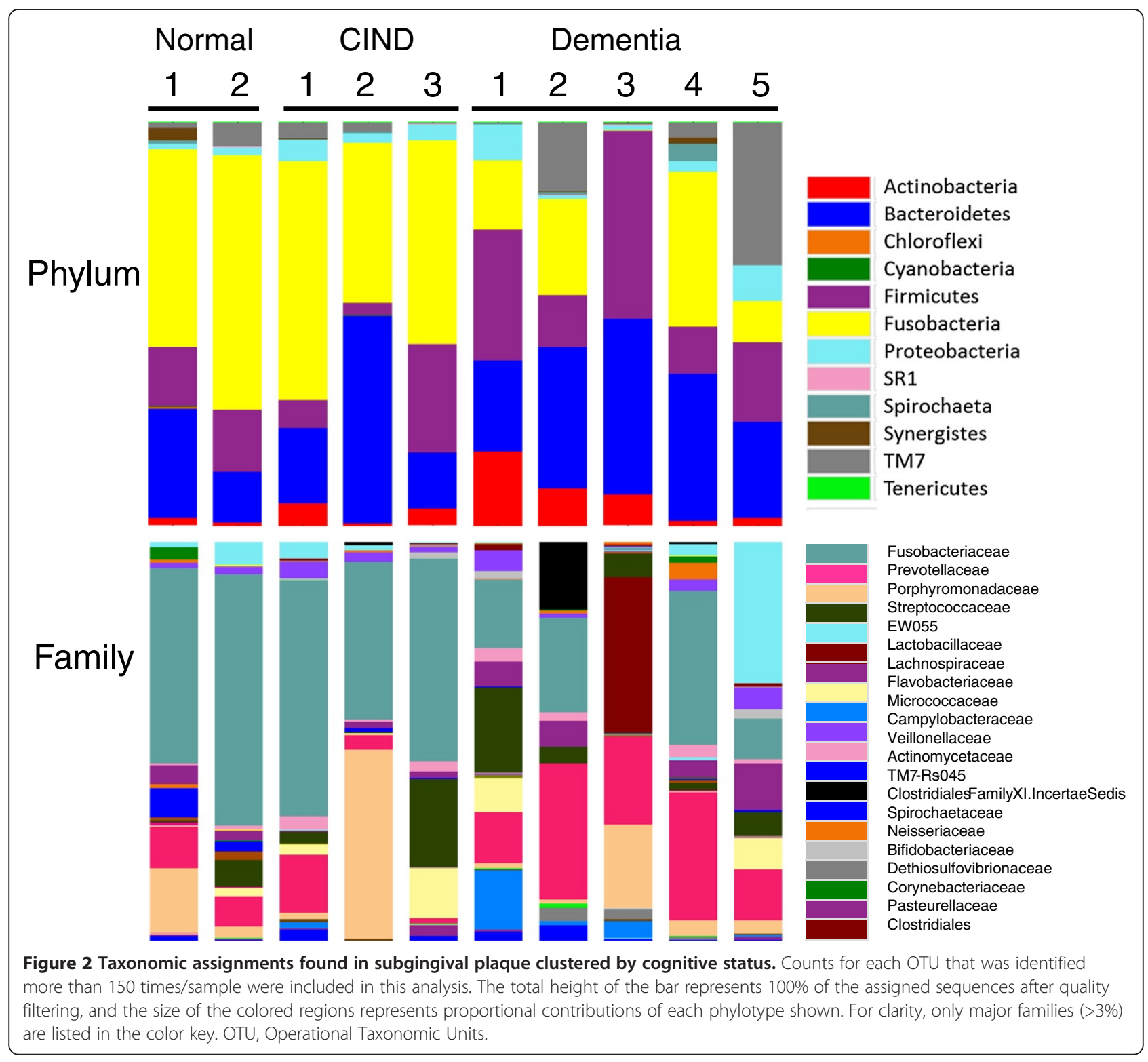

assigned to this group. There were only five phylotypes of Fusobacterium, although this was the most common genus in the entire data set.

Subroutines in QIIME were used to compute the UniFrac weighted beta diversity distance matrix among samples. Beta diversity is the measure of differences between samples in the abundance of phylotypes. Weighted UniFrac takes into account the phylogenetic distances between the OTUs, so that it captures differences not only at the level of individual phylotypes but also differences at higher taxonomic levels. The first two principal coordinates are plotted in Figure 4. The normal and CIND subjects tend to cluster in the top left of the graph and the subjects with dementia tend to be in the upper and lower right half of the graph. There is no obvious separation of normal from CIND. The first principal coordinate separates all but one of the normal/CIND subjects from the participants with dementia and the second principal coordinate provides some additional separation. The observed clustering in the non-dementia samples was due to differences in Fusobacteriaceae and Prevotellaceae. When the analysis was conducted with only Fusobacteriaceae and Prevotellaceae, the first coordinate completely separated the participants with dementia from the others. When all Fusobacteriaceae and Prevotellaceae were removed and the analyses repeated, there was no clustering of the non-dementia samples (Figure 4). Dementia versus non-dementia was the only variable that produced clustering on the principal coordinates plot. No clustering was evident for gingivitis score, age, sex, or number of teeth (data not shown). A variety of tests were used to determine whether these 


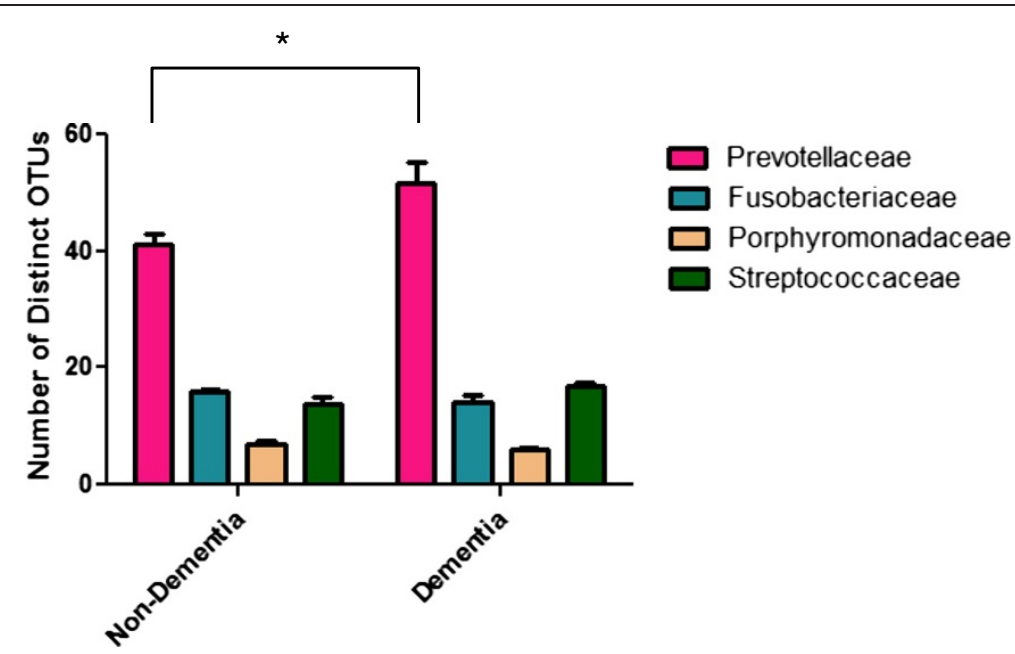

Figure 3 High number of distinct OTUs assigned to Prevotellaceae from both dementia and non-dementia samples. The number of distinct OTUs that are identified in four families that represent 2/3 of the total sequences were averaged for all samples and shown. Error bars represent standard error. Asterisk represents statistically significant difference by analysis of variance followed by Tukey's multiple comparison test $(P<0.05)$. OTU, Operational Taxonomic Units.

observed differences were statistically significant. Logistic regression analysis indicated that the levels of Fusobacterium and Prevotella were significantly different $(P<0.0018$ and $P<0.0049$, respectively) between nondementia and dementia. However, random forest analysis, a somewhat more robust test of statistical significance indicated that the observed differences did not reach statistical significance.

Samples from multiple probing depths were obtained from 5 of the 10 participants and individual analysis of paired samples at the phylum and genus levels are shown in Figure 5. In samples from four of the five participants, subgingival plaque harbored similar distributions of genera irrespective of probing depth, although the percentages of minor genera differed substantially among participants. However, for the two samples from Dementia 3, the samples from the shallower probing depth of 3 to $5 \mathrm{~mm}$ contained high levels of lactobacilli and streptococci, whereas the sample from $>5 \mathrm{~mm}$ contained a high level of undefined Porphyromonads.

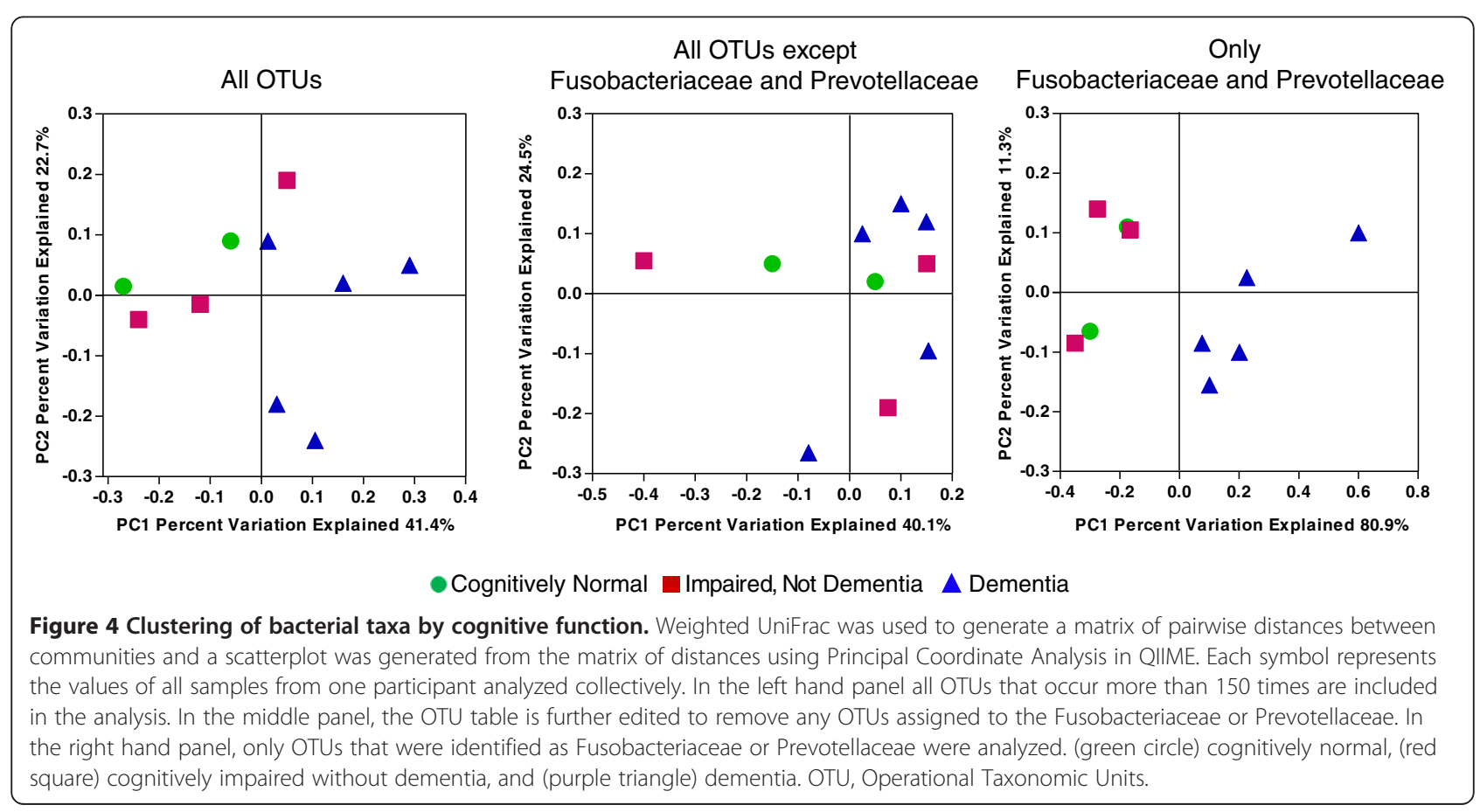




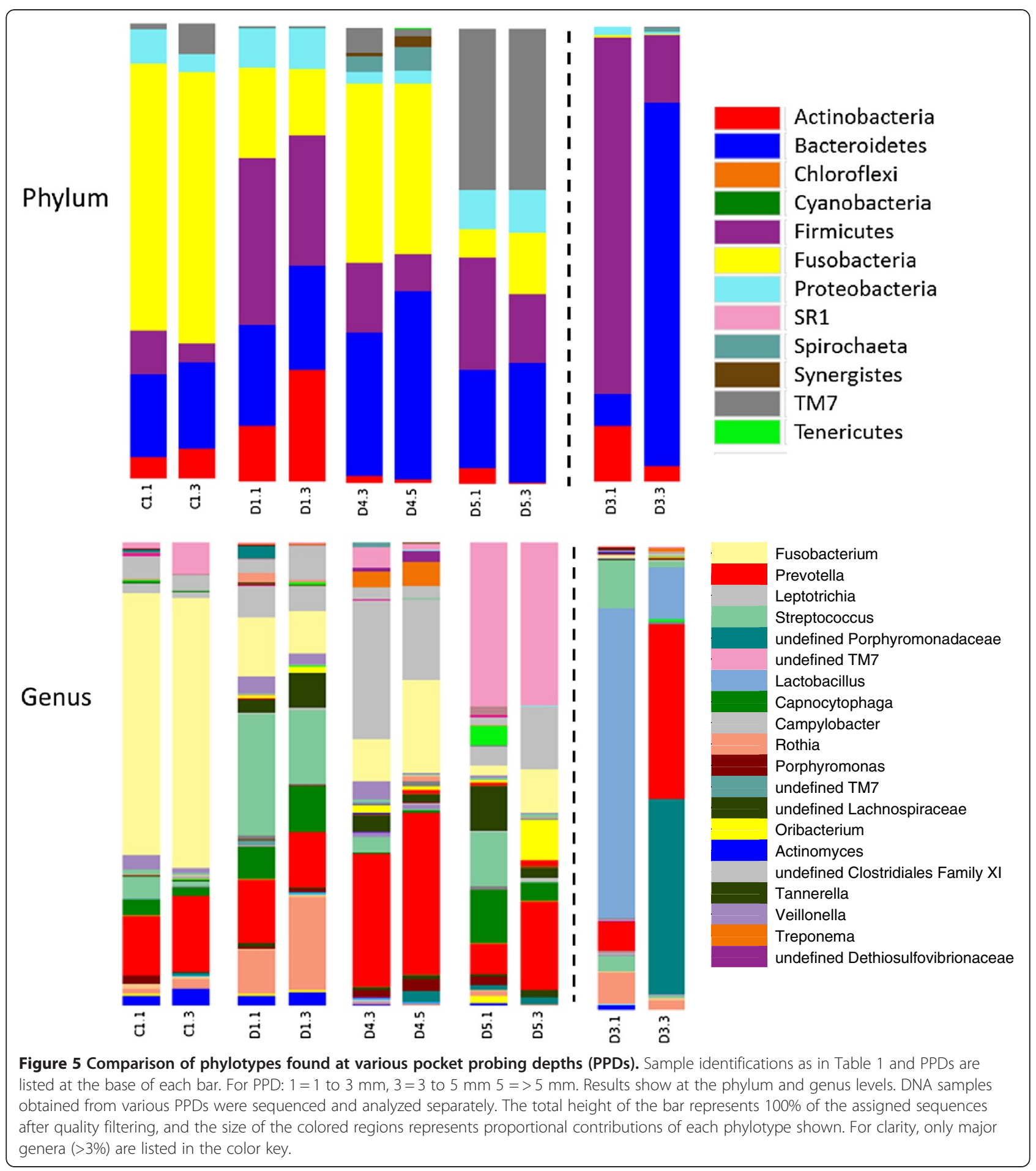

\section{Discussion}

The two purposes of this study were to develop a sample preparation and analysis pipeline to assess the oral microbiome using high throughput DNA sequencing, and to expand an ongoing study on the relationship between oral health and cognitive function in older West Virginians.
The major advantage of the Illumina platform is its capacity to generate millions of reads from each sample. Because of the relatively short read lengths, care must be used in choosing an appropriate region of the 16S RNA gene for analysis using the Illumina platform. The V3 region was selected because the primers used are the same as those used for older methods of bacterial community 
analysis, and this region had been used previously in Illumina-based analysis of microbial communities from environmental samples [27]. The region amplified in this study is longer (170 to 190 bases) than the V6 region (105 to 120 bases) [30] or the V5 region (approximately 82 bases) [18] sequenced in other studies. Using the PCR primers described in Bartram et al. [27] it was possible to run up to 12 samples per sequencing lane in this study, thereby substantially reducing the cost of the analysis. However, a challenge to using this system for microbiome analysis is the relatively short read lengths that are typically generated in a run (approximately $125 \mathrm{bp}$ ) and the lower quality of many of these reads. These disadvantages are obviated by using a paired-end sequencing approach, and successful microbiome analyses of various environmental niches [27] including the oral cavity $[18,31]$ have been documented. Furthermore, recent additions to the QIIME program have streamlined analysis of Illumina-generated data. We used the Greengenes database to identify the taxa corresponding to our sequences. About $5 \%$ of our sequences were not found in Greengenes; we believe that most of these are artifacts, but it is possible that a small number of rare OTUs could have been excluded, which limits the utility of this approach for identifying very rare phylotypes with a high level of confidence. Nevertheless, we successfully obtained millions of sequences from each sample, yielding profound details of the structure of the microbiome in subgingival plaque.

Although the main goal of this pilot study was to work out methods for obtaining high quality data and performing subsequent analysis using validated, universally available software and databases, two interesting observations were made during the phylogenetic analysis of the data. First, a very high level of Fusobacteria was found, particularly in the samples from normal and CIND participants. Fusobacteria are well-studied anaerobes that have been found with great frequency in the oral cavity using culture-independent analyses [32-35], and members of the genus Fusobacterium were previously found to be among the most commonly identified species in the oral cavities of elderly patients [34,35], particularly in association with root caries [35]. A second novel observation was that the levels of Fusobacteriaceae were lower, and that levels of Prevotellaceae were higher in samples from subjects with dementia compared to subjects without dementia. We had hundreds of taxa in our results, so by chance some of them would likely appear to be correlated with dementia. However, Prevotellaceae and Fusobacteriaceae are the two most abundant families of bacteria, and antibody levels to individual species in those families have been shown to increase to higher levels in people who develop dementia than in those who do not [13].
There are four possible explanations for the correlations between dementia and components of the microbiome: 1) the correlations are spurious due to the small sample size; 2) dementia affects the microbiome; 3) the microbiome affects dementia; and 4) a third variable affects both.

First, we acknowledge that the sample size is small and that many more subjects need to be evaluated to obtain a robust result. Whether a larger sample size will confirm these preliminary observations is an open question.

Second, it might seem self-evident that individuals with dementia have poor oral hygiene resulting from changes in diet or oral hygiene behavior, and therefore worse oral health than individuals without dementia. As expected, the participants with dementia in this study had on average, slightly more gingivitis, fewer teeth, more caries, and much higher plaque indices. However, while this is true on average, it was not always the case on an individual basis. Participant Normal 2 had poor oral health while participants Dementia 1 and Dementia 5 had relatively good oral health, albeit with fewer teeth. Participant Dementia 2 had the highest number of teeth of all those in the study. If dementia causes poor oral health, which in turn causes the changes in the microbiome, then the correlations between the directly related parameters (cognition and oral health, or oral health and the microbiome) should be higher than the correlation between the indirectly related parameters (cognition and the microbiome). Since we found the opposite, the data do not support the hypothesis that the observed differences are merely secondary effects of poor oral hygiene in subjects with dementia.

We found more Prevotella on average in the samples from participants with dementia than in the samples from participants without dementia. However, the difference was not large and the statistical significance of that finding was dependent on the statistical test used to analyze the data. The number of Prevotellaceae phylotypes was high in both groups of samples, supporting many previous studies that showed diversity in Prevotellaceae phylotypes/species in the oral cavity [36]. In addition, there was a slight but statistically significant increase in the number of distinct OTUs in the dementia samples compared to the non-dementia samples, raising the question of whether there are phylotypes in the Prevotellaceae that contribute to dementia. At the species and strain levels there are examples of specific genes that could potentially contribute to virulence within the Prevotellaceae family including genes that encode fimbrial adhesins, phospholipases, host-resistance factors, adenine-specific DNA-methyltransferase and 8-amino-7oxononanoate synthase [36,37]. Species-specific insertion sequences have also been identified [37], but whether these or other genes are disproportionately 
expressed in dementia patients and play a role in disease awaits metagenomic analyses. There were no other predominant phylotypes found in higher levels in participants with dementia compared to non-dementia, arguing against the idea that the presence of certain bacteria promotes dementia. However, the fact that higher levels of Fusobacteriaceae were found in all samples from participants without dementia suggests an alternate explanation, that perhaps certain oral bacteria provide protection against dementia, possibly by filling environmental niches that could be populated by more inflammatory microorganisms, by actively suppressing local or systemic inflammatory responses, or by producing biomolecules that are neuroprotective.

The final possibility is that both dementia and the microbiome are affected by a third variable. There is a strong genetic link to some forms of dementia, including the presence of the APOE-e4 variant of the Apolipoprotein $\mathrm{E}$ gene [38]. It is possible that the presence or absence of specific taxa could be due to genetic factors in the subject such as host immune responses, expression of adhesion molecules on host tissues that affect bacterial adherence, or other undefined factors. The relationship between human genotype and the oral microbiome needs to be studied carefully.

Sparks Stein et al. [13] found elevated levels of antibodies to Prevotella intermedia and Fusobacterium nucleatum in the blood of subjects who later developed AD. These investigators also found that subjects with Mild Cognitive Impairment (MCI), unlike AD subjects, had no differences in $P$. intermedia and F. nucleatum compared to normal subjects, but had reduced levels of antibodies to several other oral bacteria. Similarly, we found that our normal and CIND subjects did not separate based on their microbiome beta diversity and, in particular, that their Prevotellaceae and Fusobacteriaceae were similar. We hypothesize that our results can be reconciled with those of Sparks Stein et al. by predicting that subjects who will develop dementia have a leakier sub-gingival compartment resulting in increased interaction between the microbiome and the immune system, leading to higher antibody levels to the most prevalent bacteria: Fusobacterium and Prevotella. Compared to Prevotella, Fusobacteria are much less genetically diverse at the $16 \mathrm{~S}$ gene, so they might be more sensitive to elevated serum antibody levels because of less diversity of surface proteins that could serve as targets for antibodies. Thus, later in life one might predict that higher levels of antibody might reduce levels of Fusobacteria yet fail to be as effective against genetically diverse Prevotella. Alternatively, it is possible that the difference in findings for Fusobacteriaceae might be because Sparks Stein et al. were using antibodies that would differentiate strains on the basis of surface proteins while we used $16 \mathrm{~S}$ ribosomal sequences.
In summary, our results demonstrate, via high throughput DNA sequencing, that substantial inter-person variability exists in the oral microbiome of subgingival plaque. There appears to be a consistent difference in the levels of Fusobacteriaceae, and perhaps Prevotellaceae, in samples from patients who do or do not have dementia, which should be studied in more detail.

\section{Conclusions}

We have shown that high throughput DNA sequencing is an effective and inexpensive method for analyzing the microbiome of oral subgingival plaque from individual subjects. It is sensitive enough to provide a measure of the bacteria from a single sampling site. Substantial inter-person variability exists in the sub-gingival plaque microbiome, while there is generally little variation at depths ranging from 1 to $5 \mathrm{~mm}$ in an individual subject's mouth. There appears to be a consistent difference in the levels of Fusobacteriaceae, and perhaps Prevotella, in samples from patients who do or do not have dementia, which should be studied in more detail.

\section{Additional file}

Additional file 1: Comma Separated Value (.csv). Trimmed OTU Table Generated by QIIME. This is a comma separated value table that is generated by QIIME listing the number of hits for each OTU in each sample. The table has been trimmed to include all phylotypes that occur at least 150 times in the analysis.

\section{Abbreviations}

AD: Alzheimer's Disease; ANOVA: analysis of variance; bp: base pair; CIND: cognitive impairment without dementia; lgG: immunoglobulin G; MCl: Mild Cognitive Impairment; NHANES-III: Third National Health and Nutrition Examination Survey; OTU: operational taxonomic unit; PPD: pocket probing depth; QIIME: Quantitative Insights into Microbial Ecology.

\section{Competing interests}

The authors declare that they have no competing interests.

\section{Authors' contributions}

AFC aided in study design, carried out DNA isolation, amplification, and purification, performed data analysis, and helped draft the manuscript. JD wrote programming code for aligning and organizing sequences and performed data analysis. TN coordinated the study, obtained samples, performed evaluations and organized clinical data. DP, GB, and JD participated in study design and performed DNA sequencing and primary evaluation of sequencing data. RC participated in study design, in recruitment and evaluation of participants and helped draft the manuscript. BW and BP participated in the design of the study and performed analysis of data related to cognitive function and helped draft the manuscript. CC conceived of the study, participated in its design and execution, and helped to draft the manuscript. All authors read and approved the final manuscript.

\section{Acknowledgements}

This project was funded in part by the National Institute of Dental and Craniofacial Research (1R21DE016970, PI: Bei Wu) and awards from the National Center for Research Resources/National Institute of General Medical Sciences (2P20RR016477/8P20GM103434) to the WV-IDeA Network of Biomedical Research Excellence (WV-INBRE). Assistance with statistical analysis was provided by Gerald R. Hobbs and Mark V. Culp of the West Virginia University Department of Statistics. Joan Olson kindly provided DNA 
samples from the earlier study. The authors thank the study participants and their families.

\section{Author details}

'Microbiology, Immunology \& Cell Biology, School of Medicine, Robert C. Byrd Health Sciences Center, West Virginia University, P.O. Box 4622 Morgantown, WV 26506-4622, USA. ${ }^{2}$ Periodontics, School of Dentistry, Robert C. Byrd Health Sciences Center, West Virginia University, P.O. Box 9400, Morgantown, WV 26506-9400, USA. ${ }^{3}$ Department of Biochemistry and Microbiology, Robert C. Byrd Biotechnology Science Center, Joan C. Edwards School of Medicine, One John Marshall Drive, Huntington, WW 25755, USA. ${ }^{4}$ Department of Psychiatry and Behavioral Sciences, Duke University Medical Center, 2200 West Main Street, Durham, NC 27706, USA. ${ }^{5}$ School of Nursing and Global Health Institute, Duke University, 307 Trent Drive, Durham, NC 27710, USA.

Received: 28 June 2012 Accepted: 30 August 2012 Published: 21 September 2012

\section{References}

1. Kamer AR, Dasanayake AP, Craig RG, Glodzik-Sobanska L, Bry M, Leon MJ: Alzheimer's disease and peripheral infections: the possible contribution from periodontal infections, model and hypothesis. J Alzheimers Dis 2008, 13:437-449.

2. Kaye EK, Valencia A, Baba N, Spiro A, Dietrich T, Garcia Rl: Tooth loss and periodontal disease predict poor cognitive function in older men. $J \mathrm{Am}$ Geriatr Soc 2010, 58:713-718.

3. Grabe HJ, Schwahn C, Völzke H, Spitzer C, Freyberger HJ, John U, Mundt T, Biffar R, Kocher T: Tooth loss and cognitive impairment. J Clin Periodontol 2009, 36:550-557.

4. Stewart R, Hirani V: Dental health and cognitive impairment in an English national survey population. J Am Geriatr Soc 2007, 55:1410-1414.

5. Wu B, Plassman BL, Crout RJ, Liang J: Cognitive function and oral health among community-dwelling older adults. J Gerontol A Biol Sci Med Sci 2008, 63:495-500

6. Stein PS, Kryscio RJ, Desrosiers M, Donegan SJ, Gibbs MB: Tooth loss, apolipoprotein E, and decline in delayed word recall. J Dent Res 2010, 89:473-477.

7. Kamer AR, Craig RG, Pirraglia E, Dasanayake AP, Norman RG, Boylan RJ, Nehorayoff A, Glodzik L, Brys M, de Leon MJ: TNF-alpha and antibodies to periodontal bacteria discriminate between Alzheimer's disease patients and normal subjects. J Neuroimmunol 2009, 216:92-97.

8. Batty GD, Li Q, Huxley R, Zoungas S, Taylor BA, Neal B, de Galan B, Woodward M, Harrap SB, Colagiuri S, Patel A, Chalmers J, On behalf of the ADVANCE Collaborative group: Oral disease in relation to future risk of dementia and cognitive decline: prospective cohort study based on the Action in Diabetes and Vascular Disease: Preterax and Diamicron Modified-Release Controlled Evaluation (ADVANCE) trial. Eur Psychiatry 2011, epub ahead of print.

9. Miklossy J: Alzheimer's disease-a spirochetosis? Neuroreport 1993, 4:841-848.

10. Riviere GR, Riviere $\mathrm{KH}$, Smith KS: Molecular and immunological evidence of oral Treponema in the human brain and their association with Alzheimer's disease. Oral Microbiol Immunol 2002, 17:113-118.

11. Stewart R, Sabbah W, Tsakos G, D'Aiuto F, Watt RG: Oral health and cognitive function in the Third National Health and Nutrition Examination Survey (NHANES III). Psychosom Med 2008, 70:936-941.

12. Noble JM, Borrell LN, Papapanou PN, Elkind MS, Scarmeas N, Wright CB: Periodontitis is associated with cognitive impairment among older adults: analysis of NHANES-III. J Neurol Neurosurg Psychiatry 2009, 80:1206-1211.

13. Sparks SP, Steffen MJ, Smith C, Jicha G, Ebersole JL, Abner E, Dawson D III: Serum antibodies to periodontal pathogens are a risk factor for Alzheimer's disease. Alzheimers Dement 2012, 8:196-203.

14. Aas JA, Paster BJ, Stokes LN, Olsen I, Dewhirst FE: Defining the normal bacterial flora of the oral cavity. J Clin Microbiol 2005, 43:5721-5732.

15. Dewhirst FE, Chen T, Izard J, Paster BJ, Tanner AC, Yu WH, Lakshmanan A, Wade WG: The human oral microbiome. J Bacterio/ 2010, 192:5002-5017.

16. Olson JC, Cuff CF, Lukomski S, Lukomska E, Canizales Y, Wu B, Crout RJ Thomas JG, McNeil DW, Weyant RJ, Marazita ML, Paster BJ, Elliott T: Use of $16 \mathrm{~S}$ ribosomal RNA gene analyses to characterize the bacterial signature associated with poor oral health in West Virginia. BMC Oral Health 2011, 11:7.

17. Lazarevic V, Whiteson K, Gaia N, Gizard Y, Hernandez D, Farinelli L, Osteras $M$, Francois $P$, Schrenzel J: Analysis of the salivary microbiome using culture-independent techniques. J Clin Bioinforma 2012, 2:4.

18. Lazarevic V, Whiteson K, Huse S, Hernandez D, Farinelli L, Osteras M Schrenzel J, Francois P: Metagenomic study of the oral microbiota by Illumina high-throughput sequencing. J Microbiol Methods 2009, 79:266-271.

19. Keijser BJ, Zaura E, Huse SM, van der Vossen JM, Schuren FH, Montijn RC, ten Cate JM, Crielaard W: Pyrosequencing analysis of the oral microflora of healthy adults. J Dent Res 2008, 87:1016-1020.

20. Wu B, Goedereis EA, Crout RJ, Plassman BL, DiNapoli EA, McNeil DW, Wiener $M$, Boone ML, Wiener RC, Kao E, Bai L: Recruitment of rural and cognitively impaired older adults for dental research. Spec Care Dentist 2010, 30:193-199.

21. Caporaso JG, Kuczynski J, Stombaugh J, Bittinger K, Bushman FD, Costello EK, Fierer N, Pena AG, Goodrich JK, Gordon Jl, Huttley GA, Kelley ST, Knights D, Koenig JE, Ley RE, Lozupone CA, McDonald D, Muegge BD, Pirrung M, Reeder J, Sevinsky JR, Turnbaugh PJ, Walters WA, Widmann J, Yatsunenko T, Zaneveld J, Knight R: QIIME allows analysis of high-throughput community sequencing data. Nat Methods 2010, 7:335-336.

22. Centers for Disease Control and Prevention (CDC): National Center for Health Statistics (NCHS). National Health and Nutrition Examination Survey Examination Protocol. Hyattsville, MD: U.S. Department of Health and Human Services; 2001

23. Shah A, Phongsathorn V, Bielawska C, Katona C: Screening for depression among geriatric inpatients with short versions of the Geriatric Depression Scale. Int J Geriatr Psychiatry 1996, 11:915-918.

24. American Psychiatric Association: Diagnostic and Statistical Manual of Mental Disorders. Washington DC: American Psychiatric Association; 1994

25. Plassman BL, Steffens DC, Burke JR, Welsh-Bohmer KA, Newman TN, Drosdick D, Helms MJ, Potter GG, Breitner JC: Duke twins study of memory in aging in the NAS-NRC twin registry. Twin Res Hum Genet 2006, 9:950-957.

26. Plassman BL, Langa KM, McCammon RJ, Fisher GG, Potter GG, Burke JR, Steffens DC, Foster NL, Giordani B, Unverzagt FW, Welsh-Bohmer KA, Heeringa SG, Weir DR, Wallace RB: Incidence of dementia and cognitive impairment, not dementia in the United States. Ann Neurol 2011, 70:418-426

27. Bartram AK, Lynch MD, Stearns JC, Moreno-Hagelsieb G, Neufeld JD: Generation of multimillion-sequence 16S rRNA gene libraries from complex microbial communities by assembling paired-end Illumina reads. Appl Environ Microbiol 2011, 77:3846-3852.

28. R Development Core Team: $R$ : A language and environment for statistical computing. Vienna, Austria: R Foundation for Statistical Computing; 2011

29. Nadkarni MA, Browne GV, Chhour KL, Byun R, Nguyen KA, Chapple CC, Jacques NA, Hunter N: Pattern of distribution of Prevotella species/ phylotypes associated with healthy gingiva and periodontal disease. Eur J Clin Microbiol Infect Dis 2012, in press.

30. Gloor GB, Hummelen R, Macklaim JM, Dickson RJ, Fernandes AD, MacPhee $R$, Reid $G$ : Microbiome profiling by illumina sequencing of combinatorial sequence-tagged PCR products. PLoS One 2010, 5:e15406.

31. Lazarevic V, Whiteson K, Hernandez D, Francois P, Schrenzel J: Study of inter- and intra-individual variations in the salivary microbiota. $B M C$ Genomics 2010, 11:523.

32. Colombo AP, Bennet S, Cotton SL, Goodson JM, Kent R, Haffajee AD, Socransky SS, Hasturk H, Van Dyke TE, Dewhirst FE, Paster BJ: Impact of periodontal therapy on the subgingival microbiota of severe periodontitis: comparison between good responders and "refractory" subjects by the human oral microbe identification microarray (HOMIM). $J$ Periodontol 2012, in press.

33. Ahn J, Yang L, Paster BJ, Ganly I, Morris L, Pei Z, Hayes RB: Oral microbiome profiles: $16 \mathrm{~S}$ rRNA pyrosequencing and microarray assay comparison. PLoS One 2011, 6:e22788

34. Preza D, Olsen I, Willumsen T, Grinde B, Paster BJ: Diversity and sitespecificity of the oral microflora in the elderly. Eur J Clin Microbiol Infect Dis 2009, 28:1033-1040.

35. Preza D, Olsen I, Willumsen T, Boches SK, Cotton SL, Grinde B, Paster BJ: Microarray analysis of the microflora of root caries in elderly. Eur J Clin Microbiol Infect Dis 2009, 28:509-517. 
36. Alauzet C, Marchandin H, Lozniewski A: New insights into Prevotella diversity and medical microbiology. Future Microbiol 2010, 5:1695-1718.

37. Masakiyo Y, Yoshida A, Shintani Y, Takahashi Y, Ansai T, Takehara T: The identification of genes specific to Prevotella intermedia and Prevotella nigrescens using genomic subtractive hybridization. Anaerobe 2010, 16:265-269.

38. Ward A, Crean S, Mercaldi CJ, Collins JM, Boyd D, Cook MN, Arrighi HM: Prevalence of apolipoprotein E4 genotype and homozygotes (APOE e4/ 4) among patients diagnosed with Alzheimer's disease: a systematic review and meta-analysis. Neuroepidemiology 2012, 38:1-17.

doi:10.1186/2041-2223-3-19

Cite this article as: Cockburn et al:: High throughput DNA sequencing to detect differences in the subgingival plaque microbiome in elderly subjects with and without dementia. Investigative Genetics 2012 3:19.

\section{Submit your next manuscript to BioMed Central and take full advantage of:}

- Convenient online submission

- Thorough peer review

- No space constraints or color figure charges

- Immediate publication on acceptance

- Inclusion in PubMed, CAS, Scopus and Google Scholar

- Research which is freely available for redistribution 